\title{
Platelet Count and Bleeding in Patients Receiving Anticoagulant Therapy for Venous Thromboembolism: Lesson from the RIETE Registry
}

This article was published in the following Dove Press journal:

Journal of Blood Medicine

\author{
Pierpaolo Di Micco' \\ Manuel Monreal iD ${ }^{2}$ \\ 'Department of Internal Medicine and \\ Emergency Room, Ospedale Buon \\ Consiglio Fatebenefratelli, Naples, Italy; \\ ${ }^{2}$ Department of Internal Medicine, \\ Hospital Universitari Germans Trias \\ i Pujol, Universidad Autónoma de \\ Barcelona, Badalona, Barcelona, Spain
}

Correspondence: Pierpaolo Di Micco Email pdimicco@libero.it

\begin{abstract}
Major bleeding is one of the most dangerous complications for patients undergoing anticoagulant treatment for VTE. Several clinical scores have been planned to identify patients at higher risk of bleeding, and most of them take into consideration the number of platelets in particular if lower than normal. Here we report the clinical experience made with the RIETE registry concerning anticoagulant treatment in the presence of different values of platelets and their related risk of bleeding.
\end{abstract}

Keywords: thrombocytopenia, venous thromboembolism, bleeding, bleeding risk

\section{The Risk of Bleeding During Anticoagulation}

Morbidity and mortality of patients affected by venous thromboembolism (VTE) is a major health problem because it is the third cause of mortality for vascular diseases in Western countries. Moreover, VTE is also associated with a number of recurrent episodes and for this reason there are several risk factors that influence VTE recurrences as far as other complications, such as major bleeding. An appropriate clinical approach based on the knowledge of these risk factors may facilitate the use of antithrombotic therapy to identifypatients that may benefit from careful management. Early identification of clinical complications may facilitate supportive measures, thus reducing morbidity and mortality for VTE. A number of variables, identified by several articles, including age, cancer, history of bleeding, anemia, thrombocytopenia, renal insufficiency, intensity and duration of anticoagulant therapy or use of concomitant drugs, have been associated with a worse outcome in patients with VTE. ${ }^{1,2}$ However, the role of platelet count in the outcome in patients treated for VTE is still matter of discussion.

The incidence of bleeding associated with therapeutic doses of unfractionated heparin, low-molecular-weight heparin (LMWH) or vitamin K antagonists (VKAs) is around $10-17 \%$ per year for all events, $2-5 \%$ per year for major bleeding, and $0.5-1 \%$ per year for fatal bleeding. ${ }^{3}$ The reported annual occurrence of intracranial bleeding, which represents the most feared bleeding complication because of its high disability and fatality rate, is in the range of $0.2-0.4 \% .{ }^{4-6}$ Stratification of the individual risk of bleeding prior to initiation of therapy in patients with acute VTE has the potential to assist clinicians in making decisions about the proper intensity and duration of anticoagulant therapy. A number of prognostic scores have been developed with variable grades of complexity and reproducibility. ${ }^{7-10}$ Unfortunately however, none of the 
evaluated bleeding scores has an adequate accuracy and power to accurately identify VTE patients at increased risk of major bleeding.

\section{Platelet Count at Baseline and Bleeding During Anticoagulation}

Platelets count is very important before to start an antithrombotic treatment as testified by previous studies and by the presence of thrombocytopenia as one of the variables in some risk assessment tools to identify patients at risk for bleeding after a VTE. ${ }^{11,12}$ However, a confirm of this evidence was also underlined by a study that investigated 21,887 longitudinal blood counts in a multicenter cohort of 776 patients with different levels of platelets' count and found a U-shaped curve for the relationship between platelet count and risk of major bleeding. ${ }^{5}$ So after this evidence we were interested to know if such a relationship could also be found in VTE patients receiving anticoagulation.

We used the data in the RIETE (Registro Informatizado Enfermedad Trombo Embólica) registry to compare the three-month outcomes of patients with acute VTE according to their platelet count at baseline. We categorized patients as having very low- $(<80,000 / \mu \mathrm{L})$, low- $(80,000 / \mu \mathrm{L}$ to $150,000 / \mu \mathrm{L})$, normal- $(150,000 / \mu \mathrm{L}$ to $300,000 / \mu \mathrm{L})$, high$(300,000 / \mu \mathrm{L}$ to $450,000 / \mu \mathrm{L})$, or very high $(>450,000 / \mu \mathrm{L})$ platelet count at baseline, and compared their three-month outcomes in 43,078 patients with acute VTE. Of the studied population, $1.2 \%$ had very low platelet count, while $13 \%$ had low platelet count, $66 \%$ had a normal count, $17 \%$ had a high platelet count and $3.6 \%$ had a very high platelet count at baseline. No differences in the rate of VTE recurrences was found in the follow-up period, but the rate of major bleeding was different for each subgroup: 5.8\%, $2.6 \%, 1.7 \%, 2.3 \%$, and $4.6 \%$, respectively for reported groups, underlining a nearly threefold increase of bleedings for patients receiving anticoagulation with very low platelets count. This trend was respected also for the rate of fatal bleeding: $2.0 \%, 0.9 \%, 0.3 \%, 0.5 \%$, and $1.2 \%$, respectively, with a double fold increase for patients receiving anticoagulation with very low platelet count. Multivariate analysis confirmed this clinical evidence for patients with a very low platelet count and a low platelet count had an increased risk for major bleeding and fatal bleeding compared to those with a normal platelet count. Thus, we found a U-shaped relationship between platelet count and the three-month rate of major bleeding and fatal bleeding in patients with VTE. ${ }^{11,12}$

Our data confirmed that patients with a very low platelet count had a higher rate of major bleeding, $1.2 \%$ of these patients had $<80,000$ platelets $/ \mu \mathrm{L}$ and revealed that these patients had increased risk for major bleeding and fatal bleeding than those with normal platelet count. Moreover, the follow up showed in the study confirmed that this worse outcome was evident from the first week of treatment and was found both in patients initially presenting with pulmonary embolism (PE) and in those with deep vein thrombosis (DVT), and persisted after multivariate adjustment. To confirm this clinical aspect, the study testified that the increase in bleeding risk was low (less than two-fold) in patients with only mild abnormalities in platelet count, but similar to some variables (i.e., age, body weight, BMI and so on) appearing in most validated scores for predicting the risk of major bleeding. Statistical analysis on this study was based on data reported as percentage, and frequencies; data were analyzed with SPSS software (version 15, SPSS, Chicago, IL, USA), $\mathrm{p}$ was considered to be significant if its values were $<0.05$; The Fisher exact test and the odds ratio and $95 \%$ confidence intervals were calculated.

Our study had several important limitations. First, only a one-time measure of platelet counts limited reliability and clarity of reported findings. Platelet count should be considered as a dynamic monitor for a patient who is taking anticoagulants after a thrombotic event as VTE; this monitor is useful not only to evaluate the risk of bleeding per se but mainly because platelet count may be modified by several environmental conditions (e.g., comorbidities and their related therapies). Furthermore, not all causes of thrombocytopenia are present in the database of RIETE registry and with current variables almost $50 \%$ of acquired thrombocytopenias (i.e., cancerassociated thrombocytopenia, chemotherapy-associated thrombocytopenia and hypomobility-associated thrombocytopenia) may be explained as reported in Table 1. So, although the baseline test is useful there is a needful testtest variability during the follow up. In particular, attention should be given to patients receiving chemotherapy because of the possible transient thrombocytopenia, and with recent surgery that may develop transient thrombocytosis. Furthermore, platelet counts should be always confirmed by a double-check by any other means (smear/manual count), in order to avoid false thrombocytopenia that are also associated to different platelets' 
Table I Comorbidities Associated with Thrombocytopenia in Reported Studies

\begin{tabular}{|l|l|l|l|}
\hline Study & $\begin{array}{l}\text { Cancer-Associated } \\
\text { Thrombocytopenia }\end{array}$ & $\begin{array}{l}\text { Hypomobility-Associated } \\
\text { Thrombocytopenia }\end{array}$ & $\begin{array}{l}\text { Other Unidentified } \\
\text { Causes }\end{array}$ \\
\hline Di Micco et al $2012^{(12)}$ & $40 \%$ & $22 \%$ & $38 \%$ \\
Di Micco et al $2013^{(1)}$ & $41 \%$ & $19 \%$ & $40 \%$ \\
Giorgi-Pierfranceschi et al & $39 \%$ & $17 \%$ & $44 \%$ \\
$2015^{(5)}$ & & & \\
\hline
\end{tabular}

morphology. Moreover, despite our efforts to control any bias from underlying diseases, it is likely that we were unable to eliminate such bias completely. Finally, a thorough check of all type of thrombocytopenias should be associated to their severity, in particular when a major bleed has been associated to thrombocytopenia at baseline.

\section{Platelet Count and Bleeding During Anticoagulation with VKAs}

In a subsequent study, we aimed to analyze the influence of platelets in 37,000 patients receiving long-term therapy for VTE with VKAs. ${ }^{5}$ Overall, 611 patients (1.6\%) had a very low platelet count, $4006(10.8 \%)$ had a low platelet count, 25,598 (69\%) had a normal platelet count, 5,801 (15.6\%) had a high- platelet count and 984 (2.6\%) had a very high platelet count at baseline. Patients with very low or very high platelet count levels were more likely to have comorbidities such as metastatic cancer, chronic liver disease, renal insufficiency, anemia or recent major bleeding. During the course of VKA therapy (mean, 192 days), there were no differences in the duration or intensity (as measured by international normalized ratio levels) of treatment between subgroups. The rate of major bleeding was $3.6 \%, 2.1 \%, 1.9 \%, 2.1 \%$, and $3.7 \%$, respectively, and the rate of fatal bleeding was $0.98 \%, 0.17 \%, 0.29 \%$, $0.34 \%$, and $0.50 \%$, respectively. Thus, we confirmed the existence of a non-linear "U-shaped" relationship between platelet count and bleeding in VTE patients undergoing long-term VKA therapy. We also found additional differences among subgroups in the severity of bleeding, but not in time course or site of bleeding. At variance with previously reported findings, the rate of fatal bleeding was significantly higher in patients with very low platelet count, but not in those with very high platelet count. However, these data show a clinical limitation because not all causes of thrombocytopenia are present as item in the database of the RIETE registry, so whit current clinical variables only nearly a $50 \%$ of causes of acquired thrombocytopenia may be identified, as cancer associated thrombocytopenia as chemotherapy associated thrombocytopenia as hypomobility associated thrombocytopenia (Table 1). Statistical analysis on this study was based on $\chi^{2}$-test for categorical variables and $\mathrm{p}$ was considered to be significant if its values were $<0.05$; incidence rates were calculated as cumulative incidence and compared using the rate ratio.

In summary, we found a linear U-shaped curve for patients with different platelets count at the onset of VTE and a non-linear U-shaped relationship between platelet count at baseline and major bleeding in patients receiving anticoagulant therapy for VTE. Compared to other studies in this report we found an increased rate of fatal bleeding of patients with a very low platelet counts and this can find a clinical explanation because the RIETE registry frequently records patients with several comorbidities that may be associated with thrombocytopenia and that may be associated to poor outcome per se as advanced cancer; on the other hand other studies reported in the Literature have been usually focused only on the outcome of selected population with thrombocytopenia and this bias of selection may influence the different outcome with our results.

\section{Conclusion}

Our findings may be of help in identifying patients who require closer observation during anticoagulation because they are at risk of bleeding, but further dedicated studies are needed to evaluate the causality of this relationship.

\section{Ethics}

The authors certify that institutional approval is not required by their Institutions, because the intellectual property of reported data is of the RIETE registry; ethic committee that approved the RIETE registry in Fatebenefratelli Hospital of Naples was "Comitato Etico Lazio1". 


\section{Disclosure}

Professor Manuel Monreal reports grants and personal fees from Sanofi, grants from Bayer, and personal fees from Leo, during the conduct of the study. The authors report no other conflicts of interest in this work.

\section{References}

1. Monreal M. Fatal pulmonary embolism or bleeding in patients with venous thromboembolism: implications of real-life data. Thromb Res. 2019;181(Suppl 1):S6-S9.

2. Jiménez D, Bikdeli B, Quezada A, et al; RIETE Investigators. Hospital volume and outcomes for acute pulmonary embolism: multinational population based cohort study. BMJ. 2019;366:14416. doi:10.1136/ bmj.14416

3. Hlavacek P, Guo JD, Rosenblatt L, et al. Safety, effectiveness, and health care cost comparisons among elderly patients with venous thromboembolism prescribed warfarin or apixaban in the United States Medicare population. Curr Med Res Opin. 2019;35 (12):2043-2051. doi:10.1080/03007995.2019.1653067

4. Ageno W, Turpie AG. Low-molecular-weight heparin in the treatment of pulmonary embolism. Semin Vasc Surg. 2000;13(3):189-193.

5. Giorgi-Pierfranceschi M, Di Micco P, Cattabiani C, et al; RIETE Investigators. Platelet count and major bleeding in patients receiving vitamin $\mathrm{K}$ antagonists for acute venous thromboembolism, findings from real world clinical practice. Medicine (Baltimore). 2015;94(47): e1915. doi:10.1097/MD.0000000000001915
6. Yan Y-D, Zhang C, Shen L, et al. Net clinical benefit of non-vitamin $\mathrm{K}$ antagonist oral anticoagulants for venous thromboembolism prophylaxis in patients with cancer: a systematic review and trade-off analysis from 9 randomized controlled trials. Front Pharmacol. 2018;9:575. doi:10.3389/fphar.2018.00575

7. Rosenberg DJ, Press A, Fishbein J, et al. External validation of the IMPROVE Bleeding Risk Assessment Model in medical patients. Thromb Haemost. 2016;116(3):530-536. doi:10.1160/TH16-01-0003

8. Piovella C, Dalla Valle F, Trujillo-Santos J, et al; RIETE Investigators. Comparison of four scores to predict major bleeding in patients receiving anticoagulation for venous thromboembolism: findings from the RIETE registry. Intern Emerg Med. 2014;9 (8):847-852. doi:10.1007/s11739-014-1073-8

9. Nieto JA, Solano R, Trapero Iglesias N, et al; RIETE Investigators. Validation of a score for predicting fatal bleeding in patients receiving anticoagulation for venous thromboembolism. Thromb Res. 2013;132(2):175-179. doi:10.1016/j.thromres.2013.06.019

10. Fauchier L, Chaize G, Gaudin A-F, Vainchtock A, Rushton-Smith SK, Cotté F-E. Predictive ability of HAS-BLED, HEMORR2HAGES, and ATRIA bleeding risk scores in patients with atrial fibrillation. A French nationwide cross-sectional study. Int J Cardiol. 2016;217:85-91. doi:10.1016/j.ijcard.2016.04.173

11. Di Micco P, Ruiz-Giménez N, Nieto JA, et al; RIETE Investigators. Platelet count and outcome in patients with acute venous thromboembolism. Thromb Haemost. 2013;110(5):1025-1034. doi:10.1160/TH13-04-0352

12. Di Micco P, Fontanella A, Falvo N, et al; RIETE Investigators. Natural history of patients developing thrombocytopenia while receiving anticoagulant therapy for venous thromboembolism. Int Angiol. 2012;31(1):92-95.
Journal of Blood Medicine

\section{Publish your work in this journal}

The Journal of Blood Medicine is an international, peer-reviewed, open access, online journal publishing laboratory, experimental and clinical aspects of all aspect pertaining to blood based medicine including but not limited to: Transfusion Medicine; Blood collection, Donor issues, Transmittable diseases, and Blood banking logistics; Immunohematology; Artificial and alternative blood based

\section{Dovepress}

therapeutics; Hematology; Biotechnology/nanotechnology of blood related medicine; Legal aspects of blood medicine; Historical perspectives. The manuscript management system is completely online and includes a very quick and fair peer-review system. Visit http://www.dovepress.com/testimonials.php to read real quotes from published authors. 